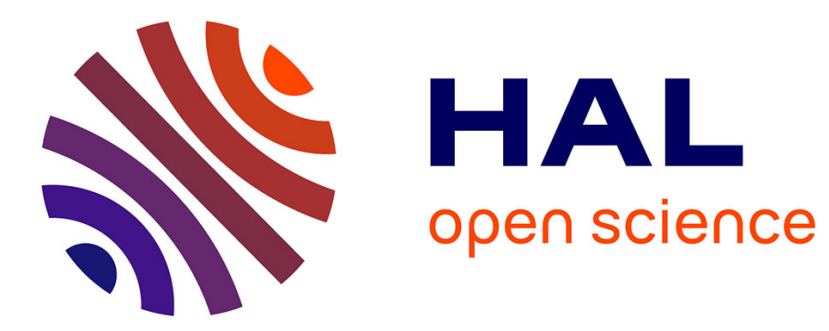

\title{
Surge Compression for Improved Fault Location Accuracy in Full Transient-Based Methods
}

\author{
Andréa Cozza, Shao-Yin He, Yan-Zhao Xie
}

\section{To cite this version:}

Andréa Cozza, Shao-Yin He, Yan-Zhao Xie. Surge Compression for Improved Fault Location Accuracy in Full Transient-Based Methods. IEEE Sensors Journal, 2021, 21 (2), pp.995 - 1008. 10.1109/JSEN.2020.2989202 . hal-02546433

\section{HAL Id: hal-02546433 \\ https://hal.science/hal-02546433}

Submitted on 17 Apr 2020

HAL is a multi-disciplinary open access archive for the deposit and dissemination of scientific research documents, whether they are published or not. The documents may come from teaching and research institutions in France or abroad, or from public or private research centers.
L'archive ouverte pluridisciplinaire HAL, est destinée au dépôt et à la diffusion de documents scientifiques de niveau recherche, publiés ou non, émanant des établissements d'enseignement et de recherche français ou étrangers, des laboratoires publics ou privés. 


\title{
Surge Compression for Improved Fault Location Accuracy in Full Transient-Based Methods
}

\author{
Andrea Cozza, Senior Member, IEEE, Shao-yin He, Member, IEEE Yan-zhao Xie, Senior Member, IEEE
}

\begin{abstract}
This paper proves that the accuracy of singleended fault location techniques based on the correlation of fault transients in power lines can be significantly improved by inversefiltering transients first. The reason for this improved accuracy is found in the reduction of the duration of the fault surge signal, thus increasing the sensitivity of fault-location metrics to the fault position. Reported results show that by using the proposed filtering strategy, faster sampling rates systematically and significantly improve the location accuracy, as opposed to the case when no filter is applied, for which virtually no improvement is observed. Because of its higher spatial selectivity, the proposed fault-location technique required the development of a new synchronization method, which ensures a precise transient synchronization without the need for high-speed sampling. It is concluded that by jointly using the proposed transient filtering and synchronization solutions, correlation-based fault location can be significantly improved even when using sampling rates as low as $100 \mathrm{kHz}$. Extensive numerical simulations for a threephase transmission line confirm an improvement exceeding one order of magnitude in the fault location accuracy, for both lowand high-impedance faults.
\end{abstract}

Index Terms-Fault location, power transmission line, location accuracy, synchronization, correlation, inverse filters, fault surge.

\section{LIST OF SYMBOLS}

$T_{o}$ sampling offset between measured and reference transients, with respect to the transient inception

$T$ round-trip probe-fault propagation delay

$T_{e}$ residual synchronization error after synchronization

$\delta t \quad$ sampling period

$T_{W}$ recorded transient window

\section{INTRODUCTION}

$\mathbf{T}$ HE protection of power-transmission lines relies on the ability to locate (and isolate) faults, before they give rise to power outages [1]. Location accuracy is therefore a fundamental figure of merit when comparing fault-location methods. Among the various solutions developed over the past decades, traveling-wave methods (TWM) exploit transient signals caused by the surge pulse generated by shunt faults. These transients are typically recorded by means of single or multiple probes installed at the terminations of a line. Upon

(Corresponding author : A. Cozza.).

S.-Y. He, Y.-Z. Xie, are with the State Key Laboratory of Electrical Insulation and Power Equipment, School of Electrical Engineering, Xi'an Jiaotong University, Xi'an 710049, China (email: shaoyin.he@xjtu.edu.cn; yzxie@xjtu.edu.cn).

A. Cozza is with the Group of Electrical Engineering - Paris (GeePs), CentraleSupelec, Univ. Paris-Sud, Université Paris-Saclay, Sorbonne Universites, UPMC Univ Paris 06, 3 \& 11 rue Joliot-Curie, Plateau de Moulon 91192 Gif-sur-Yvette CEDEX, France (email: andrea.cozza@ieee.org). propagating along a line, the fault surge undergoes multiple interactions with discontinuities along a line ${ }^{1}$, giving way to a series of echoes that bear a strong dependence on the fault position. TWM estimate the fault distance from a line termination by computing the delay between the first two occurrences of the fault surge, appearing in the early portion of transients, in a fashion similar to time-domain reflectometry [2]-[11].

Another class of TWM exploits transients more thoroughly, by including later echoes and potentially the full transient [4], [12]-[20], resulting in a more accurate fault location according to [13], [14], [20], [21]. The reasons for their higher location accuracy were discussed in [22]. These full transientbased methods (TBM) typically locate a fault by comparing a measured transient with reference transients expected for faults at different positions: the fault position is identified as the one bearing the highest similarity between measured and reference transients. These reference data are most of the time based on numerical simulations, but can also make use of experimental data [13]. TBM are found throughout the literature to implement either direct transient correlation [13], [14], [20] or indirect comparison involving normalized projections. This last case has been mostly given consideration under the electromagnetic time-reversal (EMTR) technique [15], [17], [21], [23], [24]. In fact, it was proven in [16] that EMTR mimics a correlation metric.

Both TBM approaches have been shown to effectively estimate a fault position from its full transient, but the use of an a posteriori normalization in projection-based TBM was proven to be susceptible to location biases, particularly so in presence of non-negligible propagation losses [25]. For this reason, this paper will focus only on the case of transient correlation, but the ideas here discussed can also be applied to projection-based methods, such as EMTR.

The location metric considered in this paper therefore is the correlation coefficient

$$
\rho(\hat{L}, L)=\left[v_{m}[k ; L] \mid v_{m}[k ; \hat{L}]\right] / \sqrt{\mathcal{E}_{m}(L) \mathcal{E}_{m}(\hat{L})}
$$

between a measured voltage ${ }^{2}$ transient $v_{m}[k ; L]$ generated by the actual fault at a position $L$, and reference transients $v_{m}[k ; \hat{L}]$ for multiple tested positions $\hat{L}$. Both transients are sampled with a time-step $\delta t$, with $k$ the index of the samples;

\footnotetext{
${ }^{1}$ including junctions, line terminations and the fault

${ }^{2}$ alternatively, current transients could also be chosen
} 
they will also be assumed to be high-passed filtered, in order to remove $\mathrm{DC}$ and nominal $\mathrm{AC}$ contributions. In (1)

$$
\left[v_{m}[k ; L] \mid v_{m}[k ; \hat{L}]\right]=\sum_{k=1}^{N} v_{m}[k ; L] v_{m}[k ; \hat{L}]
$$

represents the projection between the two transients, with $N=T_{W} / \delta t$ the total number of samples recorded over a time window $T_{W}$, while

$$
\mathcal{E}_{m}(L)=\left[v_{m}[k ; L] \mid v_{m}[k ; L]\right]
$$

is the signal energy of a transient.

The main goal of this paper is to prove that the accuracy with which faults are located in TBM can be very significantly improved by pre-filtering the transients before computing the location metric. This paper provides clear evidence that TBM, as currently implemented, do not take full advantage of the availability of high-speed sampled transients. As recalled in Sec. II, fault surge signals behave as step functions, thus resulting in a strong overlapping between successive echoes of the fault surge signal. Sec. III presents the case for the use of inverse filters aimed at compressing the duration (or time support) of the surge signal in the recorded transients, in order to reduce the superposition between echoes and therefore emphasize differences between measured and reference transients. In particular, it is proven that the location accuracy of standard TBM does not improve with higher sampling rates. Using the proposed surge-compression scheme, a more than tenfold improvement in the location accuracy is demonstrated, even with sampling rates as low as $100 \mathrm{kHz}$, and steadily improving with the sampling rate.

Because of the significantly higher sensitivity to a fault position for surge-compressed transients, a very precise transient synchronization becomes necessary. Secs. IV and V argue that the standard threshold-based synchronization is insufficient, and that high-speed sampling rates become necessary in order to provide a still sub-optimal accuracy. An alternative synchronization, using a model-based estimation of the delay of the rising front of the fault surge is presented, and proven to significantly improve the location accuracy, without relying on high-speed sampling. These tools are applied in Sec. VI to numerical simulations of a three-phase overhead transmission line, covering a number of fault configurations, confirming the significant improvement in the accuracy of fault location.

These results prove that faults can be located with a higher accuracy than currently possible with TBM, without increasing either the complexity of the post-processing currently applied in TBM, or requiring higher sampling rates. The most practical impact could be expected for lines with high-frequency propagation losses: in this case a higher sampling rate may paradoxically reduce the location accuracy, as proven in [25]. It is therefore of practical interest to be able to increase the location accuracy without relying on higher sampling rates, a standard solution adopted in TWM [14], [20], [26]. Moreover, in legacy protection relay systems transients may be sampled at rates well below $1 \mathrm{MHz}$ [13], [20], [27], [28], limiting the ability to accurately locate faults with other methods.

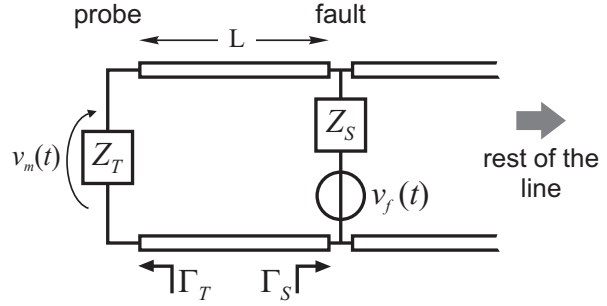

Fig. 1. Single-phase line model considered in the definition of the transient model (7). $\Gamma_{T}$ and $\Gamma_{S}$ represent the reflection coefficients of the transformer and the shunt fault, respectively.

\section{TRANSIENT MODEL}

A single-phase power transmission line is first considered, representing, e.g., underground/submarine power coaxial cables. The line is assumed to be connected at both ends to power transformers. With reference to Fig. 1, a shunt fault of impedance $Z_{S}$, at a distance $L$ from the left end, is represented by means of its Thevenin equivalent circuit, generating a surge signal $v_{f}(t)$ approximately described by a step function [1], [2]. This surge signal gives rise to traveling waves propagating away from the fault along the sections at the left and right of the fault, propagating along each section till they interact with discontinuities such as junctions and terminations. Given that this kind of faults typically present impedances by far smaller than the characteristic impedance of the line [29], the two sections will be regarded as severed in practice, with no signal propagating across the fault ${ }^{3}$. The fault surge will be reflected upon interacting with power transformers, which are expected to present, at frequencies above a few $\mathrm{kHz}$, an input impedance $Z_{T}$ with modulus much larger than the characteristic impedance of the line, thus resulting in a reflection coefficient $\Gamma_{T} \simeq 1$. The reflection coefficient for the shunt fault is rather expected to be $\Gamma_{S} \simeq-1$.

The appearance of fault transient signals $v_{m}(t)$ is monitored by means of a probe on the busbar of the power transformers, or using contact-less probes as suggested in [30]. This transient can be expressed as a series of echoes of the fault surge signal $v_{f}(t)$, each arriving at the probe after a propagation delay $T=2 L / v$, where $v$ is the propagation speed along the line, i.e.,

$$
v_{m}(t ; L)=\mathrm{e}^{-\alpha L} \sum_{n=0}^{\infty} \Gamma^{n} v_{f}\left(t-(n+1 / 2) T-T_{o}\right)
$$

with $\Gamma$ the line transmissivity

$$
\Gamma=\Gamma_{S} \Gamma_{T} \mathrm{e}^{-2 \alpha L},
$$

modeling the relative change in amplitude between two subsequent surge echoes, with $\alpha$ the attenuation constant of the line. The index $n$ represents the order or rank of each surge echo contributing to the transient recorded by the probe, with each echo arriving with a delay $T$ with respect to the previous echo. The amplitude of each incoming echo therefore decreases with their order of arrival. The term $(n+1 / 2) T$ models the

\footnotetext{
${ }^{3}$ high-impedance faults will be discussed in Sec. VI, for the case of threephase lines
} 
propagation delay between the instant when the fault occurs and the time of arrival at the probe.

The term $\exp (-\alpha L)$ at the beginning of (4) will be dropped hereafter, since it does not affect the shape of the transient but only its overall amplitude; on the other hand, propagation losses are taken into account by the line transmissivity (5). The propagation is assumed to be weakly dispersive, for the sake of simplicity, consistently with the idea pursued in this paper to avoid using very-high sampling rates, e.g., higher than $1 \mathrm{MHz}$. Dispersive propagation is not dealt with in the present work and will be developed in a future work, as discussed in Sec. VII. The main goal of this paper is to prove the importance of applying inverse filtering to transients in order to fully benefit from the availability of high sampling rates, while keeping fault location algorithms simple.

In practice, transients are sampled asynchronously. Hence, the transient inception is unlikely to occur at the time of one of the samples. The delay $T_{o} \in[0, \delta t)$ models this sampling offset in (4). It will be shown in Sec. V that the random nature of this delay plays a fundamental role in the accuracy of TBM seeking surge compression.

Fault surge signals are here modeled as step functions with a finite rise time $T_{r}$, following an exponential front

$$
v_{f}(t)=V_{o}\left(1-\mathrm{e}^{-t / T r}\right) u(t)
$$

with $u(t)$ the unit-step function. Fault surges are often approximated as unit-step functions, where the rise time is negligible, but it is sensible to expect a finite rise time, due to the inevitable inertia in any real system. A non-negligible rise time has indeed been argued in the literature, with typical values of $T_{r}$ reported to vary between 1 and $100 \mu \mathrm{s}$ [31]-[35].

Given that in real-life applications signals are sampled, a discrete-time description of (4) will be adopted

$$
v_{m}[k ; L]=\sum_{n=0}^{\infty} \Gamma^{n} v_{f}\left(k \delta t-(n+1 / 2) T-T_{o}\right),
$$

consistently with (2). The discrete nature of recorded transients, though apparently trivial at first sight, has important practical consequences when it comes to the synchronization of the transients, as discussed in Sec. IV.

This simplified transient model, illustrated in Fig. 2(a), is at the basis of all results discussed in Secs. III to V. The same model will serve for generating the fault transient and reference transients for tested fault positions. In the latter case $T_{o}=0$, since reference transients are typically generated by means of numerical simulations, and can therefore be expected, with no loss of generality, to be synchronized to the fault transient inception. More complex fault transients will be considered in Sec. VI, for the case of a three-phase transmission line.

\section{SURGE COMPRESSION}

When applied to the class of transients (7), the correlation drops off only when the transient for a tested fault position $\hat{L}$ becomes significantly different from the measured one, for a fault at a distance $L$. A rapid loss of correlation as soon as $\hat{L}$ drifts away from $L$ is a desirable feature, since it
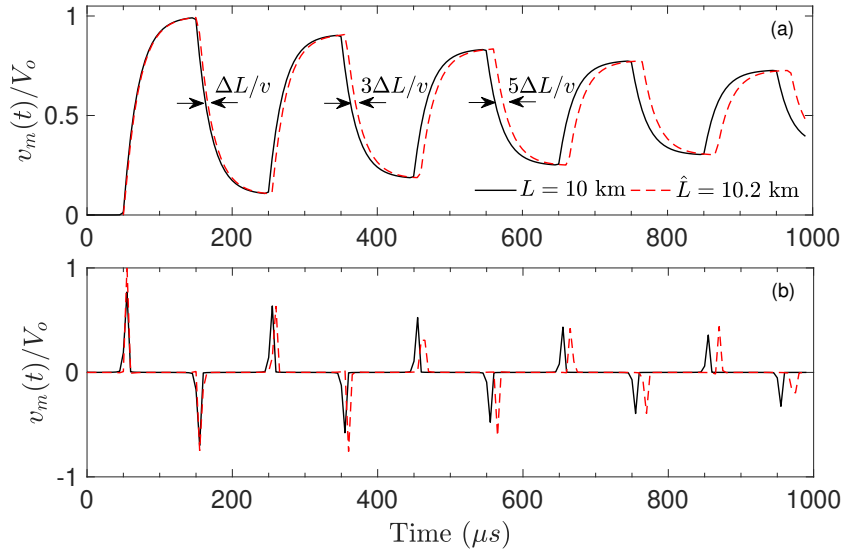

Fig. 2. Comparison between transients expected for shunt faults at a distance of 10 and $10.2 \mathrm{~km}$ along a single-phase line as in Fig. 1, for a line transmissivity $\Gamma=-0.9$ and a fault surge described by: (a) an exponential step and (b) a Kronecker delta function, resulting in a correlation equal to 0.96 and 0.4 , respectively. Each echo of the fault surge is offset by an increasing delay $n \Delta L / v$, with $n$ the order of arrival of the echo.

would pinpoint the fault position more precisely. This section focuses on how to magnify these differences and thus make correlation-based fault location more accurate.

The example in Fig. 2(a) shows fault transients obtained from (7) for faults at 10 and $10.2 \mathrm{~km}$ distances, for a propagation speed $v=2.99 \times 10^{8} \mathrm{~m} / \mathrm{s}$ and a line transmissivity $\Gamma=-0.9$, following a step surge with a rise time $T_{r}=20 \mu \mathrm{s}$, observed over a window $T_{W}=1 \mathrm{~ms}$. These transients look very similar: their correlation is about 0.96. Their similarity stems from their being generated by a superposition of step functions, each with an infinite time support. Successive echoes alternatively change in sign for a shunt fault and their interference results in a sequence of pulses with a duration roughly equal to $T=2 L / v$ each. Fig. 2(a) illustrates how differences would appear only for sufficiently large values of $\Delta L / L$, with $\Delta L=\hat{L}-L$, since in this case the overlapping between the two transients would be approximatively reduced by a factor $\Delta L / L$. It is also clear from Fig. 2(a) that the mismatch between the two transients increases for later echoes. This simple observation explains the reason why recording fault transients over a longer time window can lead to improved spatial resolution [36].

The similarity between the measured and tested transients would decrease more rapidly if a fault surge had a shorter duration. As a consequence, correlation would also drop off closer to the actual fault position, thus improving the location accuracy. Such a scenario is found in Fig. 2(b), where the fault surge is now assumed to be a Kronecker delta function. Given its very compact time support, location errors would result in this case in a stronger mismatch between measured and reference transients, resulting in a faster loss of correlation, now evaluated at 0.4 , thus dramatically reduced with respect to the correlation 0.96 obtained for the transients in Fig. 2(a). As opposed to the case of step-like surge in Fig. 2(a), later echoes in Fig. 2(b) no longer overlap, therefore making it possible to estimate more accurately the fault distance $L$, as discussed in Sec. III-B. 


\section{A. Inverse filtering}

In practice, the fault surge cannot be controlled, but an equivalent effect can be achieved by post-processing actual transients. Ideally, transients such as those in Fig. 2(a) should be converted into those seen in Fig. 2(b).

Such a procedure can be defined by first noticing that fault transients described by (7) can be expressed as a convolution product $^{4}$

$$
v_{m}[k]=h[k] * v_{\delta}[k]
$$

where $h[k]=v_{f}[k] / V_{o}$, while

$$
v_{\delta}[k]=V_{o} \sum_{n=0}^{\infty} \Gamma^{n} \delta\left[k-\left((n+1 / 2) T-T_{o}\right) / \delta t\right]
$$

is the transient that would be observed if the fault surge were a Kronecker delta function $\delta[k]$. The function $h[k]$ represents the impulse response of the equivalent filter distorting $v_{\delta}[k]$ into $v_{m}[k]$.

Therefore, the ideal transient $v_{\delta}[k]$ can be retrieved by compensating the distortion introduced by the surge signal, $h[k]$. This operation corresponds to a deconvolution and can be carried out by first computing the z-transform $H(z)$ of $h[k]$, which corresponds to the exponential-step surge described in (6). $H(z)$ can be shown to be [37, Sec. 3.3]

$$
H(z)=\left[\left(1-z^{-1}\right)\left(1-z^{-1} \mathrm{e}^{-\delta t / T_{r}}\right)\right]^{-1},
$$

by noticing that (6) can also in turn be expressed as the convolution of a decaying exponential and a unit-step (or Heaviside) function.

A simple approach to deconvolution is the use of an inverse filter, which requires the sampled transient $v_{m}[k]$ to be fed as the input signal of a filter with a transfer function $H^{-1}(z)$. Expressing $H^{-1}(z)$ in terms of delay units $z^{-1}$

$$
H^{-1}(z)=1-z^{-1}\left(1+\mathrm{e}^{-\delta t / T_{r}}\right)+z^{-2} \mathrm{e}^{-\delta t / T_{r}}
$$

shows that this inverse filter has a finite impulse response, or FIR, and can therefore be directly applied to the transient samples $v_{m}[k]$, yielding as output the signal

$\hat{v}_{\delta}[k]=v_{m}[k]-\left(1+\mathrm{e}^{-\delta t / T_{r}}\right) v_{m}[k-1]+\mathrm{e}^{-\delta t / T_{r}} v_{m}[k-2]$,

expected to approximate the sequence of Kronecker delta functions $v_{\delta}[k]$ defined in (9).

The filter (11) requires knowing the rise time $T_{r}$ of the fault surge. Sec. IV-B shows that it is indeed possible to obtain an accurate estimate of this parameter, as a by-product of a novel synchronization procedure described therein. Nevertheless, two approximations of (12) free of this constraint can be considered. The first is found in case $\exp \left(-\delta t / T_{r}\right) \ll 1$, i.e., for $\delta t>2.3 T_{r}$, in which case

$$
\hat{v}_{\delta}[k] \simeq v_{m}[k]-v_{m}[k-1],
$$

i.e., a first-order time difference ${ }^{5}$, corresponding to a lowspeed sampling, which would then approximate a unit-step

\footnotetext{
${ }^{4}$ the dependence of the transient voltage from the fault distance $L$ or $\hat{L}$ is dropped for the sake of compactness

5 the numerical equivalent of a continuous time derivative
}

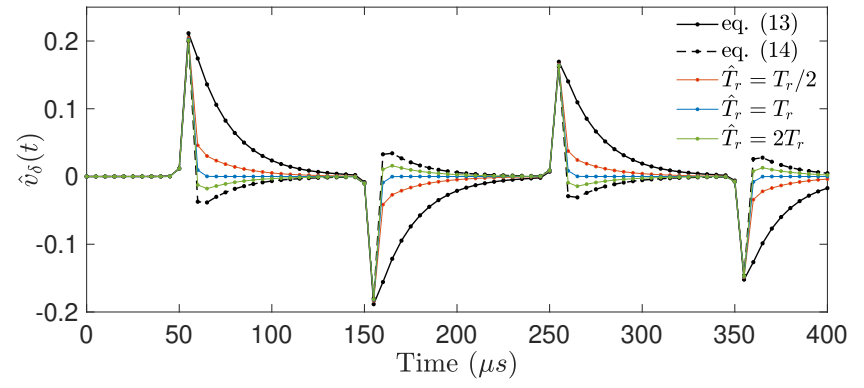

Fig. 3. Surge compression by inverse-filtering the transient in Fig. 2(a), for several choices of the estimated rise time $\hat{T}_{r}$.

function. For a rise time $T_{r}=20 \mu \mathrm{s}$, this condition would be fulfilled for a sampling rate below $20 \mathrm{kHz}$. Conversely, for high-speed sampling, with $\delta t \ll T_{r}$, (12) converges to

$$
\hat{v}_{\delta}[k] \simeq v_{m}[k]-2 v_{m}[k-1]+v_{m}[k-2],
$$

i.e., a second-order time difference. In this case, the sampling rate would need to reach at least $500 \mathrm{kHz}$.

Fig. 3 illustrates how the proposed filters operate, when applying them to the transient in Fig. 2(a), for a fault at a distance $L=10 \mathrm{~km}$, for different estimates $\hat{T}_{r}$ of the surge rise time. When (12) is applied with knowledge of the exact value of the surge rise time, i.e., $\hat{T}_{r}=T_{r}$ the transients are reduce to a sequence of Kronecker delta functions, precisely indicating the time of occurrence of the first surge and subsequent echoes. The significant reduction in the time support of each echo is seen to be weakly sensitive to inaccuracies in $\hat{T}_{r}$ : using half or twice the actual value of $T_{r}$ still results in short pulses indicating the time of arrival of each echo, followed by an exponential tail that would significantly overlap with successive echoes only for faults at distances $L \lesssim v T_{r}$, i.e., less than $6 \mathrm{~km}$ for the example at hand.

The first- and second-order derivative approximations of (12) are also used in Fig. 3. The low-speed sampling approximation (13) features a longer residual exponential tail, while the high-speed approximation (14) overcorrects the surge exponential step. Both significantly reduce the time support of the surge echoes, even with no knowledge of $T_{r}$, with (12) the most effective.

\section{B. Correlation function}

The effects of inverse filters on transient correlation can be appreciated in Fig. 4, where the correlation (1) is computed between a transient for a fault at a distance $L=10 \mathrm{~km}$ and reference transients for faults within $500 \mathrm{~m}$ from the fault. All transients were generated from the transient model (7) and assumed to be perfectly synchronized at their inception time. Three sampling periods were considered, equal to 1,2 and $5 \mu \mathrm{s}$.

The results show that for unfiltered transients increasing the sampling frequency has virtually no impact on the correlation function. Measuring the spatial resolution as the span covered by a correlation higher than 0.9 yields a resolution of $\pm 359 \mathrm{~m}$ around the fault, for all three sampling rates. Similar results are 


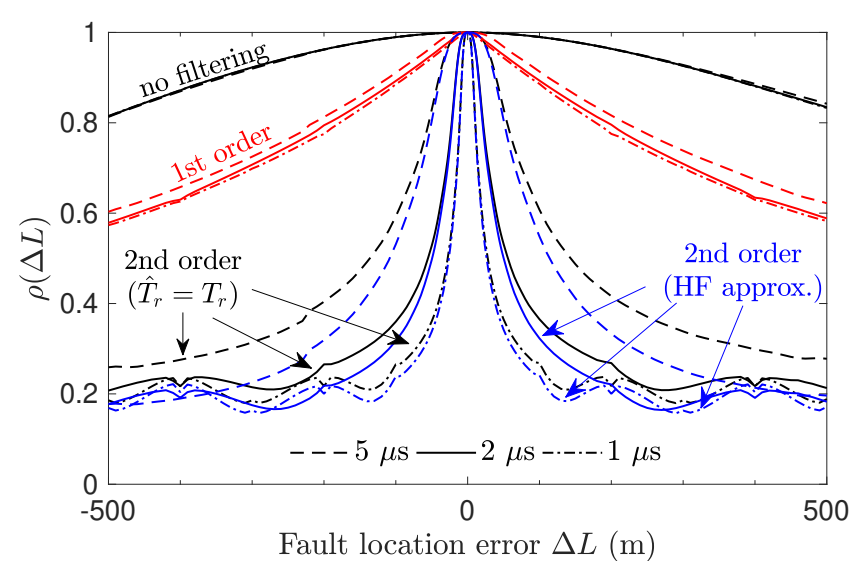

Fig. 4. Transient correlation for different filtering choices, for three sampling periods.

found in the literature for TBM, featuring a spatial resolution that can exceed $\pm 1 \mathrm{~km}$, even for sampling rates higher than $1 \mathrm{MHz}$ [24], [36].

Switching to the first-order derivative filter (13) the spatial resolution increases to $\pm 87 \mathrm{~m}$ when sampling at $\delta t=5 \mu \mathrm{s}$ and $\pm 115 \mathrm{~m}$ for $1 \mu \mathrm{s}$, thus with the location accuracy now partially improving with higher sampling rates.

Only when applying second-order filters the location accuracy fully benefits from a faster sampling rate. In case $T_{r}$ is known, the resolution is now reduced to $\pm 40 \mathrm{~m}$ from the fault, when sampling at $\delta t=5 \mu \mathrm{s}$, and passes to $\pm 16 \mathrm{~m}$ and $\pm 8 \mathrm{~m}$, when sampling at $2 \mu \mathrm{s}$ and $1 \mu \mathrm{s}$, respectively. It can be noticed how the resolution span scales linearly with $\delta t$. An almost 50-fold improvement is therefore observed in the spatial resolution with respect to the unfiltered case, when sampling at $\delta t=1 \mu \mathrm{s}$.

Surprisingly, the high-frequency approximation (14) provides a slightly better performance: this result can be understood on the basis of the over-compensation observed in Fig. 3, where each Kronecker delta function is followed by an exponential tail of opposite sign. For the other filters where each echo does not locally change sign, the correlation can decrease only when the overlapping of the actual and reference transients decreases. Hence, the high-frequency approximation (14) hastens the loss of correlation when testing transients not corresponding to the actual fault position, without requiring any estimate of the surge rise time. It can be noticed how (14) closely approximates the results for $\hat{T}_{r}=T_{r}$ for $\delta t \leq 2 \mu \mathrm{s}$, as expected from the condition $\delta t \ll T_{r}$ discussed in Sec. III-A.

For transients with a surge ideally compressed into a Kronecker delta function, as in Fig. 2(b), subsequent echoes would present no overlap as soon as they are separated by a sample period $\delta t$, i.e., for a location error $\Delta L>v \delta t / 2 n$, with $n$ the order of arrival of the surge echoes. This property indicates that when using second-order filters, the spatial resolution no longer depends on the fault distance from the probe, as opposed to the case of unfiltered transients, which were argued, at the beginning of this section, to require nonnegligible values of $\Delta L / L$ to operate. These properties are confirmed in Fig. 5, where the correlation is shown for a

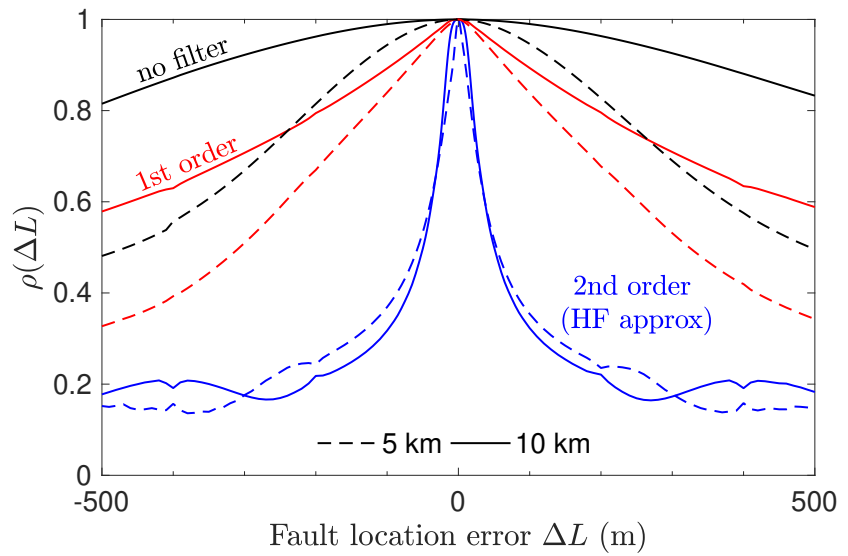

Fig. 5. Impact on the location accuracy of the fault distance, for different filtering choices. Transients sampled at $\delta t=1 \mu \mathrm{s}$.

fault at 5 and $10 \mathrm{~km}$ distance, for a line with $\Gamma=-0.9$. It can be appreciated how, for a second-order filter, location accuracy is hardly affected by a change in the fault distance. Conversely, with unfiltered transients the spatial resolution is indeed degraded for a larger fault distance, with the correlation function for $L=10 \mathrm{~km}$ stretching over a span roughly twice as large as for the case $L=5 \mathrm{~km}$.

It can be concluded that correlation-based fault location greatly benefits from the use of second-order inverse filters, resulting in a surge ideally compressed into a Kronecker delta function, yielding correlation functions more tightly bound to the fault position. Therefore, the rest of the paper will only discuss comparisons between results obtained with unfiltered transients and those processed by means of second-order filters.

\section{Line losses}

As observed in Fig. 2, surge echoes arriving later at the probe come with a higher sensitivity to location errors, thus narrowing the correlation function closer to the fault position. In case of significant transmission losses, these later echoes would be attenuated, thus less affecting the computation of the correlation function. It is therefore important to understand in what measure losses could hamper the expected improvement in fault location accuracy.

Fig. 6 shows the correlation functions obtained with the transient model (7), when testing positions close to a fault 10 $\mathrm{km}$ away from the probe. When the modulus of the transmissivity $\Gamma$, as defined in (5), decreases, the spatial resolution also decreases, i.e., the correlation function spreads across a wider region around the fault by a similar factor for both unfiltered and surge-compressed transients. The latter case has a significantly higher resolution, by a factor going from 22 to 37 , as the line losses increase. Even for relatively high losses, when $\Gamma=-0.5$, the spatial resolution ${ }^{6}$, is found to be about $67 \mathrm{~m}$ when using a second-order inverse filter, while it exceeds $2 \mathrm{~km}$ when directly computing the correlation between two transients. This property of improving the location accuracy

\footnotetext{
${ }^{6}$ setting again a threshold for $\rho=0.9$
} 


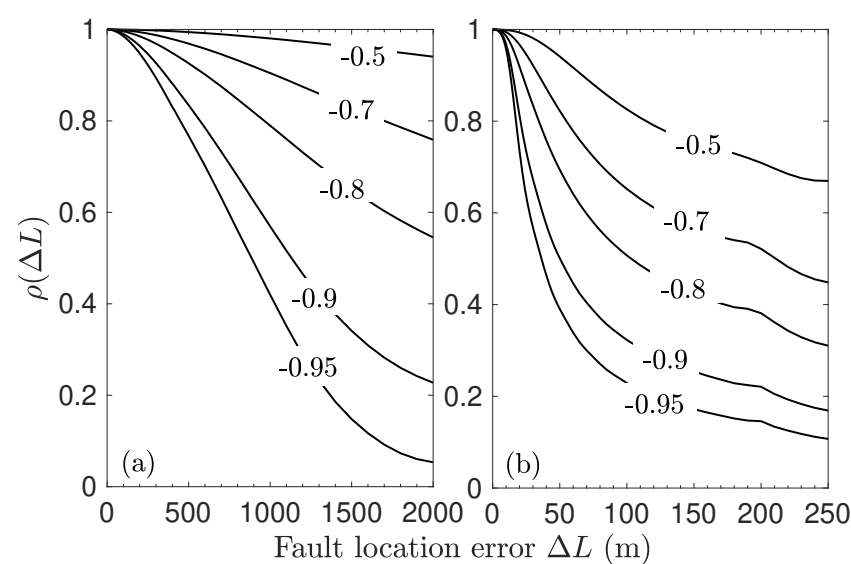

Fig. 6. Correlation function for a fault at $10 \mathrm{~km}$, using: (a) no filter and (b) the second-order filter (high-frequency approximation) in (14), for different values of the transmissivity $\Gamma$ given in (5) and a sampling period $\delta t=2 \mu \mathrm{s}$. An ideal synchronization is assumed.

as losses increase is also confirmed in Sec. VI, for the case of high-impedance faults.

\section{TRANSIENT SYNCHRONIZATION}

The measured and reference transients need to be synchronized before computing their correlation coefficient, in order to accurately estimate their similarity (overlapping): even identical signals, if subject to an offset, would result in a loss of correlation. The risk of potential systematic errors due to inaccurate synchronization dramatically increases with inverse filters, since they are designed to ideally reduce the surge echoes to Kronecker delta functions: the shorter their time support, the likelier for an offset to lead to a significant loss of overlapping and an inaccurate assessment of the similarity between the transients, ultimately resulting in location errors, as proven in Sec. V.

The need for synchronization in TBM is not to be confused with the synchronization used in multiple-ended TWM, where multiple transients are recorded at different locations along a line. In this case the synchronization requires to timestamp the transients, typically through GPS or equivalent time-reference systems, in order to estimate the propagation delay between the fault and the recording devices and thus the fault position. In this context, unsynchronized TWM schemes have been

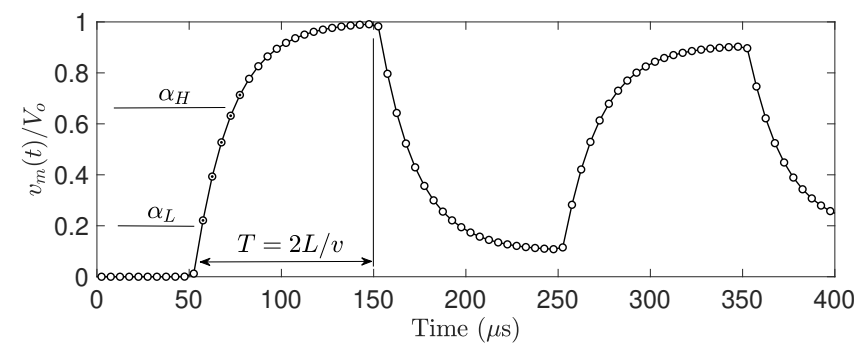

Fig. 7. Relevant parameters for the transient synchronization strategies discussed in Sec. IV. The low and high thresholds identify the samples (marked by a dot) used in the surge-parameter estimation. proposed [28], [38], dispensing with the need to timestamp transients.

In TBM a single-ended recording is commonly used, and the synchronization is no longer needed for estimating the fault position, but rather in order to accurately overlap measured and reference transients before computing their similarity [20]. No timestamp is required in this case: the alternative to transient synchronization in TBM would require computing their cross-correlation function, which would be a clearly more computationally expensive solution.

The synchronization between the transients must be ensured before they are inverse-filtered. This point can be understood by noticing that once a surge is compressed into a Kronecker delta function, only time offsets larger than a sampling step $\delta t$ can be detected, whereas the smoother and slower evolution of fault surges as those in Fig. 7 better lends itself to accurate estimates of their relative offsets.

This section discusses two synchronization strategies: threshold triggering, found throughout the literature [9], [12][14], and a novel method based on the estimation of the parameters describing the rising front of the fault surge. Their relative performance will be tested against sampling offset and period in Sec. V.

\section{A. Threshold triggering}

As discussed in [12]-[14], a simple and effective solution to transient synchronization is to apply threshold triggering. A threshold level $\alpha_{L}$ is defined, as a fraction of a transient's peak amplitude (cf. Fig. 7). The sample $n^{\star}$ for which the transient first becomes larger than the threshold is identified such that

$$
v_{m}[k ; L]<\alpha_{L} V_{o} \quad \forall k<n^{\star},
$$

with $V_{o}$ the surge amplitude. This operation is applied to both measured and reference transients, resulting in the samples $n_{m}^{\star}$ and $n_{r}^{\star}$, respectively. These are synchronized by considering their offset versions $v_{m}\left[k-n_{m}^{\star} ; L\right]$ and $v_{m}\left[k-n_{r}^{\star} ; \hat{L}\right]$.

From this definition it is clear that triggering does not attempt to estimate the delay between the two transients, but rather relies on the idea of aligning the two surge fronts by identifying the instant when the threshold is crossed. This kind of approach would be correct in a continuous-time, noiseless description. In practice, sampled transients go by a discrete-time description, which is inherently limited in its time resolution by the sampling period $\delta t$, as illustrated in Fig. 8. The effects of this limitation will be apparent in Sec. $\mathrm{V}$, when discussing the impact of sampling offset.

\section{B. Surge-parameter estimation}

A threshold-based synchronization can only compensate integer multiples of the sampling period $\delta t$. Therefore, sampling offsets cannot be corrected, unless by requiring a smaller $\delta t$, i.e., by increasing the sampling frequency. Fig. 8 defines the residual synchronization error $T_{e}$ observed after the threshold synchronization. An alternative solution is presented here, which does not call for an increase of the sampling frequency.

One way of estimating $T_{e}$ would be to fit (6) to the rising front of a transient. This would also yield estimates of the 


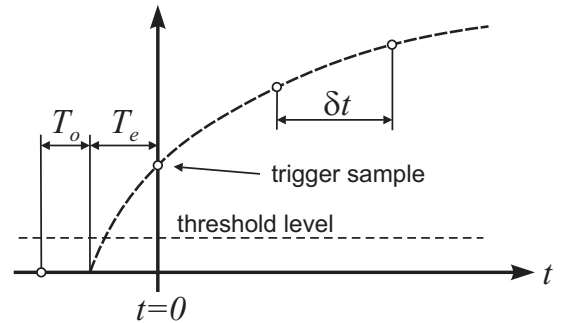

Fig. 8. Fault transient after threshold-based synchronization. The sampling offset $T_{o}$ results in a residual synchronization error $T_{e}$, estimated and corrected by the surge-based synchronization.

surge rise time $T_{r}$ and the surge amplitude $V_{o}$. Least-square methods can be used to this end, but the surge parameters $T_{r}$ and $T_{e}$ are non-linearly related to the surge samples; such configurations are well-known to give rise to computational issues in curve fitting, with results highly sensitive to the initial estimates used in the optimization procedure [39, sec. 9].

This issue can be avoided by working in two steps. First, $K$ samples from the rising front of the fault surge, identified by low and high thresholds $\alpha_{L}$ and $\alpha_{H}$ (cf. Fig. 7), are fitted by a polynomial of degree $N<K$

$$
\min _{\left\{p_{n}\right\}}\left\|v_{m}(t)-\sum_{n=0}^{N} p_{n} t^{n}\right\|,
$$

with $\|\cdot\|$ the $L_{2}$ norm, i.e., using a least-square optimization. This operation now involves a linear curve-fitting problem. It is then possible to relate the polynomial coefficients $\left\{p_{n}\right\}$ to the surge parameters, by computing the coefficients of the Taylor series of (6), now including a delay $T_{e}$

$$
V_{o}\left(1-\mathrm{e}^{-\left(t-T_{e}\right) / T_{r}}\right)=\sum_{n=0}^{N} p_{n} t^{n}
$$

from which

$$
\begin{aligned}
& p_{0} \simeq-\frac{V_{o}}{2}\left(x^{2}+2 x\right) \\
& p_{1} \simeq-\frac{V_{o}}{2 T_{r}}\left(x^{2}+2 x+2\right) \\
& p_{2} \simeq-\frac{p_{1}}{2 T_{r}}
\end{aligned}
$$

valid for $|x|<1$, where $x=T_{e} / T_{r}$, which is verified as soon as $\delta t<T_{r}$.

From (18), the rise time is found as

$$
\hat{T}_{r} \simeq-\frac{p_{1}}{2 p_{2}} .
$$

Setting $\alpha=p_{0} / p_{2}$ and $\beta=p_{1} / p_{2}$, the set of equations (18) lead to

$$
T_{e}^{2}+\beta T_{e}+\alpha=0,
$$

from which the estimator $\hat{T}_{e}$ is found as the negative root, since the transient inception necessarily occurs before its crossing the synchronization threshold, as visible in Fig. 8. $V_{o}$ can also be similarly found from

$$
\hat{V}_{o} \simeq p_{1} \hat{T}_{r}\left(1+\hat{T}_{e} / \hat{T}_{r}\right)^{-1}
$$
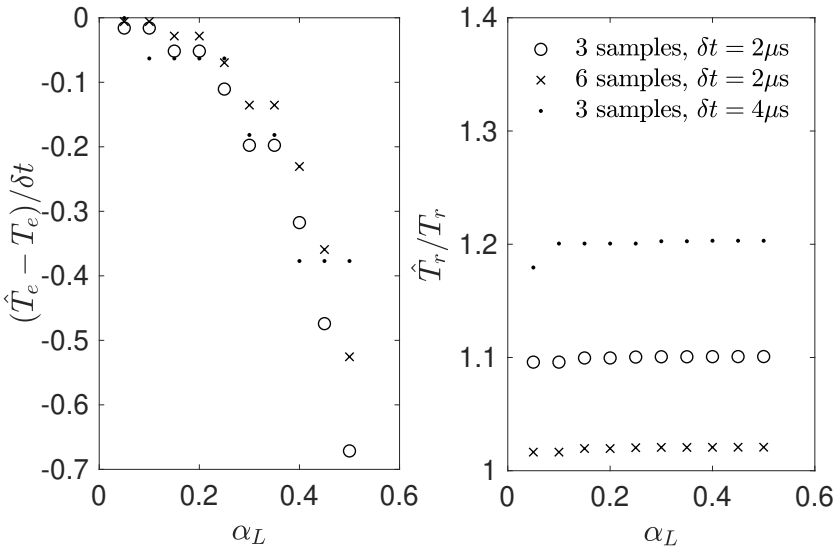

Fig. 9. Accuracy of the estimates of the residual synchronization error $T_{e}$ and rise time $T_{r}$, versus the threshold level $\alpha_{L}$ and the number of available surge samples.

and could be of interest in order to estimate the amplitude of the surge, in case its peak value is not fully attained, and therefore measurable, as happens for faults at close distance, where $T \lesssim 3 T_{r}$, i.e., $L \lesssim 1.5 v T_{r}$, roughly less than $10 \mathrm{~km}$.

The optimal choice of $N$ is a classic issue in regression problems. We refer the interested Reader to [39, sec. 1.4] for more details. The results shown in the rest of the paper are based on the choice $N=3$, which limits overfitting issues while ensuring a good estimate of coefficients $p_{0}$ to $p_{2}$ upon which the estimators are based.

The accuracy of the proposed procedure was studied by applying it to the transient shown in Fig. 7, based on a surge front with $T_{r}=20 \mu \mathrm{s}$ and an offset delay $T_{o}=0.5 \delta t$. A limited number of samples was used, of which the first was set by means of a variable low threshold $\alpha_{L} \in[0.05,0.5]$. Two different sampling periods $\delta t$ were considered, equal to 2 and $4 \mu \mathrm{s}$. Fig. 9 shows the results obtained, with the delay $T_{e}$ estimated within a fraction of $\delta t$ even when using only three samples, with errors increasing when the initial part of the surge is cut off by relatively large values of $\alpha_{L}$. The sampling period appears to have a relatively minor impact. The rise time was estimated within less than a $20 \%$ error with three samples, with the accuracy rapidly improving when more are available; the portion of the surge from which originate the samples has practically no bearing on its accuracy.

\section{Delay compensation}

Once the residual synchronization error $T_{e}$ between the two transients is estimated, the reference transient $v_{m}[k ; \hat{L}]$ needs to be resampled at sampling instants $k \delta t-T_{e}$, in order to obtain the synchronized transient $v_{m}^{\prime}[k ; \hat{L}]=v_{m}\left(k \delta t-T_{e} ; \hat{L}\right)$. This operation can be carried out by interpolating the original samples, obtained at sampling instants $k \delta t$. An effective choice is using Whittaker-Shannon interpolation [37]

$$
v_{m}^{\prime}[k ; \hat{L}]=\sum_{n} v_{m}[n ; \hat{L}] s_{w}\left(k-n-T_{e} / \delta t\right)
$$

using a windowed sinc interpolator $s_{w}(x)=w(x) \operatorname{sinc}(\pi x)$, where $w(x)$ can be any even real window function; Hamming 


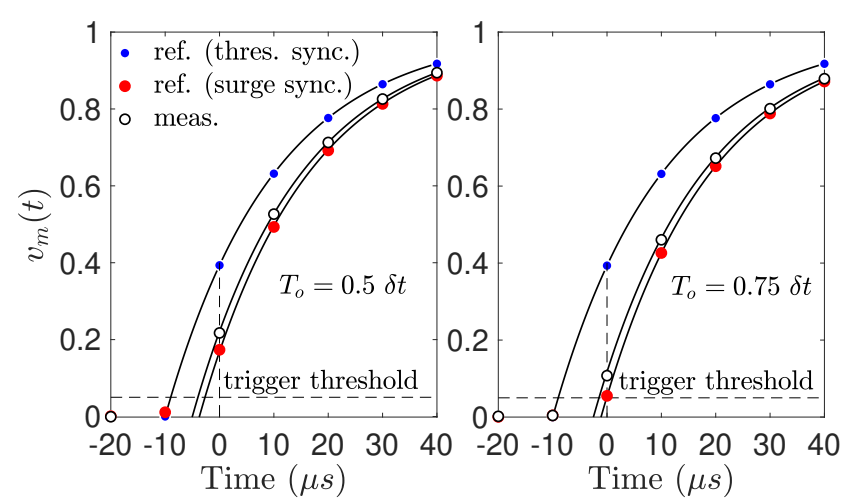

Fig. 10. Synchronization between measured and reference transients for two values of sampling offset $T_{o}$, when using a threshold-based approach and the proposed surge-front parameter estimation, as discussed in Sec. IV. Solid black lines represent the continuous transients, given as reference. Results obtained for a surge rise time $T_{r}=20 \mu \mathrm{s}$ and $\delta t=10 \mu \mathrm{s}$.

window was used in this paper. The sum is limited to the available samples.

\section{SAMPLING OFFSET}

This section compares how the two synchronization procedures discussed in the previous section cope with sampling offset and its impact on fault location using surge-compressed transients.

The effectiveness of the two synchronization procedures described in the previous section is compared in Fig. 10, for two cases of sampling offset. The measured and reference transients are identical but for a sampling offset, so an ideal synchronization would result in perfectly overlapping curves. It can be observed how the sampling offset modifies the way samples are distributed along the transients, which leads, for the case of threshold-triggering, to an imprecise synchronization. A relatively slow sampling rate, with $\delta t=10 \mu \mathrm{s}$, was

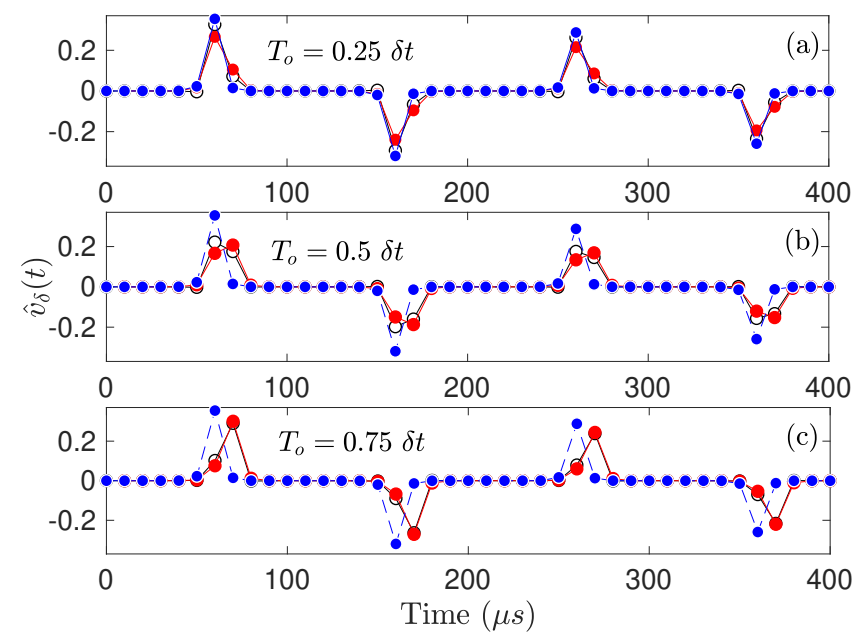

Fig. 11. The same transients from Fig. 10 after undergoing surge compression using a second-order inverse-filter, for the two synchronization strategies. Three different values of sampling offset $T_{o}$ are considered. Refer to Fig. 10 for the legend.

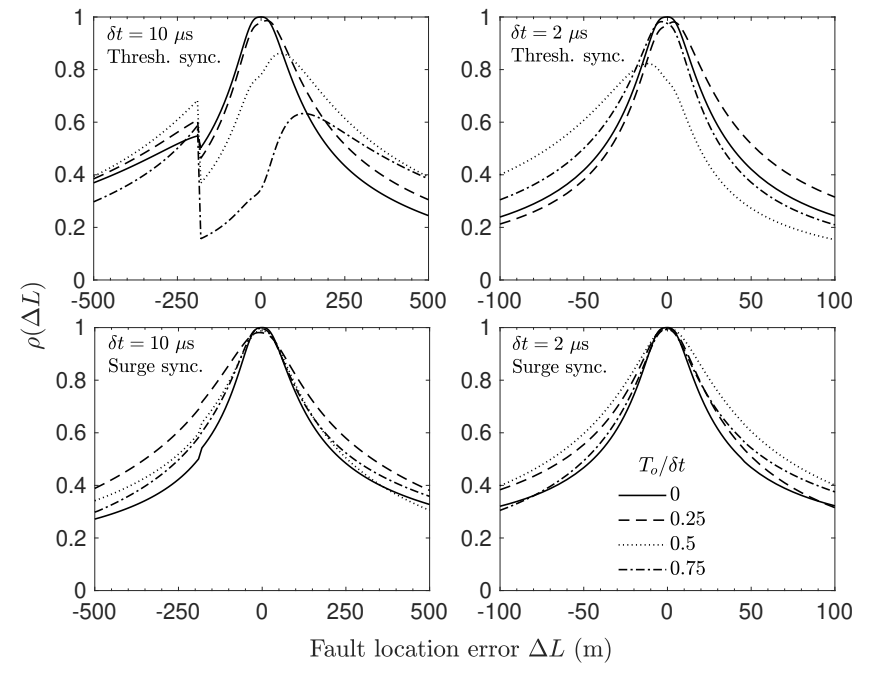

Fig. 12. Impact of sampling offset on the correlation function when using threshold-based synchronization (top row) and surge-based synchronization (bottom row). Two sampling periods are considered: $10 \mu \mathrm{s}$ (left column) and $2 \mu \mathrm{s}$ (right column). Results for $\left[\alpha_{L}, \alpha_{H}\right]=[0.05,0.7]$.

chosen in order to prove the effectiveness of the proposed synchronization strategy. The proposed synchronization approach, based on estimating the surge-front parameters, is shown to significantly reduce the impact of the sampling offset. Both approaches were applied with a low threshold equal to 0.05, with the surge-front parameters estimated from five samples, using a third-order polynomial regression (cf. Sec. IV-B).

Fig. 11 shows the surge-compressed version of these transients, after having applied the second-order inverse filter (12), assuming an accurate estimate of the surge rise time, for three values of the sampling offset. The threshold-based synchronization (blue dashed curves) is unable to compensate for increasing sampling offsets, resulting in a significant loss of overlapping, even for otherwise identical transients. When using the proposed synchronization strategy (red solid curves), this issue is significantly reduced.

The results in Fig. 12 show how synchronization errors affect the correlation, and therefore fault location. For thresholdbased synchronization, when choosing a sampling period $\delta t=10 \mu \mathrm{s}$, the correlation at the fault position decreases in case of a sampling offset, reaching a local peak at a position different from the fault's. The fact that the correlation does not reach a peak equal to one is a clear indication that the transients are not overlapped. It is worth stressing that such an issue would not be identified if the results of correlation were normalized to their peak value a posteriori, as otherwise done in projection-based TBM (cf. Sec. I). The impact of sampling offsets appears to be reduced, but not avoided, by using a significantly higher sampling rate with $\delta t=2 \mu \mathrm{s}$.

Conversely, the results obtained with the proposed synchronization approach are much less affected by sampling offsets, even when using lower sampling rates, proving its robustness. The main effect is a change in the spatial resolution, which is caused by the fact that the offset in the sampled instants correspond to a changing lower threshold $\alpha_{L}$, thus affecting 
the accuracy of the surge-based synchronization (cf. Sec. IV-B).

\section{Application to A THREE-PHASE Line}

The results presented so far were based on the transient model described in Sec. II, which only holds for single-phase configurations and low-impedance faults. When it comes to multiconductor lines, such as in bipolar HVDC lines and three-phase transmission lines, a fault should be expected to generate more complex transients, no longer presenting a regular sequence of scaled echoes. The increased complexity comes from the fact that, apart for the case where all phases where shunted to the ground, signals impinging onto the fault would only be partially reflected by it and would then propagate beyond it, giving rise to multiple series of echoes involving the two sections of the line before and after the fault. Moreover, multiconductor lines support multiple propagation modes, typically displaying non-negligible differences in their propagation speeds, as well as interacting with a fault in distinct ways, as discussed in Sec. VI-C.

In spite of these differences, the idea of surge compression still applies, since fault surge signals are still described by step-like functions [1], [29]. The main goal of this section is therefore to verify whether the improvements previously predicted for a single-phase line are also confirmed for multiconductor transmission lines.

To this end, the three-phase line shown in Fig. 13 is studied in this section, by means of numerical simulations. This line corresponds to a $10 \mathrm{kV}$ overhead power transmission line commonly employed in China. The line conductors have a radius equal to $15 \mathrm{~mm}$ and are made of aluminium. The threephase line is terminated at both ends by power transformers, modelled by termination impedances $Z_{T}=10 \mathrm{k} \Omega$ [1] applied to each conductor, while the shunt fault has an impedance $Z_{S}$, as shown in Fig. 13(a). The fault surge $v_{f}(t)$ is again described by (6), with a rise time $T_{r}$ : its impact on location accuracy is studied in Sec. VI-D. Fault transients are sampled at the left end of the line, for each phase, for a duration $T_{W}=1 \mathrm{~ms}$. EMTP-RV simulations were set up using a constant-parameter description based on the line cross-section described in Fig.

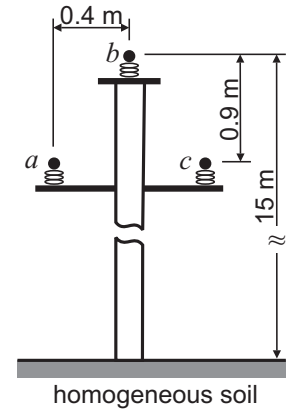

(a)

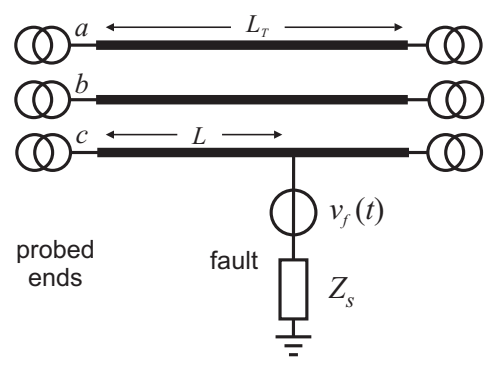

(b)
Fig. 13. The $10 \mathrm{kV}$ three-phase line studied in Sec. VI: (a) line cross section; (b) line configuration, with a single-phase to ground fault at a distance $L$ at phase $c$. The ends of each phase are connected to a power transformer, with an equivalent input impedance equal to $10 \mathrm{k} \Omega$.

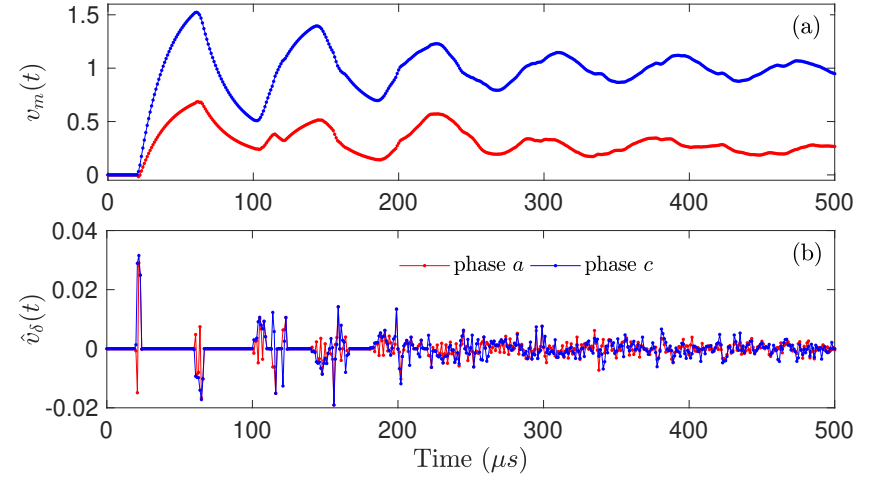

Fig. 14. Transients recorded at the left-end terminations of phases $a$ and $c$, sampled at a $1 \mathrm{MHz}$ rate, for a $10 \Omega \mathrm{PG}$ fault between phase $c$ and the ground: (a) unfiltered transients, (b) surge-compressed transients, applying the second-order filter (12).

13(a), for a soil conductivity of $10 \mathrm{mS} / \mathrm{m}$, with a $2 \Omega / \mathrm{km}$ per-unit-length resistance.

The line considered has a length $L_{T}=20 \mathrm{~km}$, with a fault at a distance $L$. Different fault types and shunt impedances $Z_{S}$ are considered in Secs. VI-A and VI-C, highlighting how the location accuracy of the proposed scheme intimately depends on the modal mix and propagation excited by each fault.

The transients recorded for the actual fault position at $L$ and those for test positions $\hat{L}$ were synchronized applying the procedure introduced in Sec. IV-B. This step was shown in $\mathrm{Sec}$. V to be fundamental in order to avoid systematic location errors caused by inevitable sampling offsets.

\section{A. Single-phase-to-ground fault}

We first focus on a single-phase-to-ground (PG) fault, since it represents the most likely configuration found in practice [29, Sec.3]; the fault will be applied to phase $c$ throughout the entire section. The fault distance is first set at $L=6 \mathrm{~km}$, with an impedance equal to $10 \Omega$ : differences observed for faults at other positions are discussed in Sec. VI-B, while other kinds of faults are discussed in Sec. VI-C. The fault surge is assumed to have a rise time $T_{r}=20 \mu \mathrm{s}$, chosen as an intermediate value in the range 1-100 $\mu$ s reported in the literature [31]-[35]; Sec. VI-D presents results for other values of $T_{r}$.

Two examples of transients are shown in Fig. 14(a), as recorded at the phases $a$ and $c$, sampled at a $1 \mathrm{MHz}$ sampling frequency. After applying the second-order filter (12) these transients present compressed surge echoes, as shown in Fig. 14(b).

A total of 25 transients were simulated, acting as the reference transients, each for a different tested fault location $\hat{L}$, over a span of $\pm 1 \mathrm{~km}$ around the actual fault position $L=6 \mathrm{~km}$. The resulting correlation function is shown in Fig. 15 , computed for three different sampling periods, from 1 to $5 \mu \mathrm{s}$. For each case the correlation function was computed for: a) the unfiltered measured and reference transients; b) after applying the first-order derivative filter (13) and c) after applying the second-order filter (12).

These results confirm that increasing the sampling frequency has no impact on the spatial resolution when using 


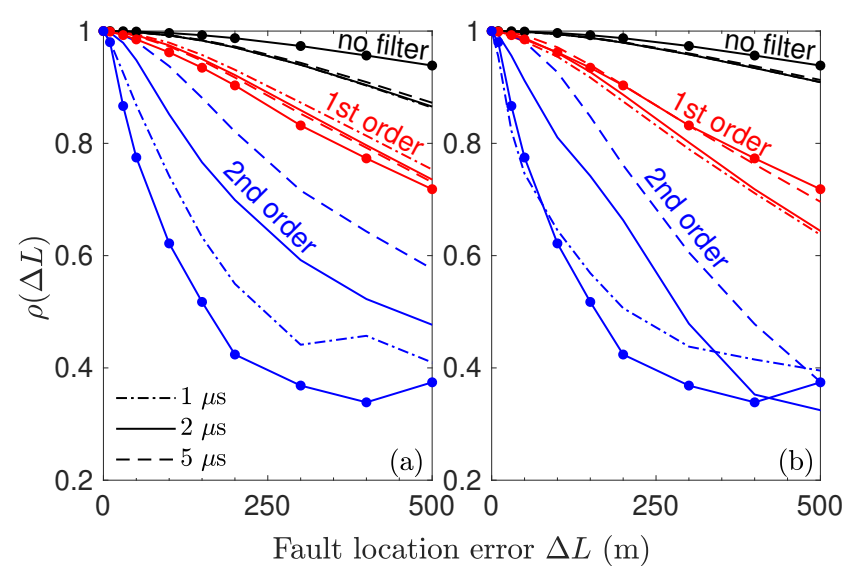

Fig. 15. Correlation function for the three-phase line in Fig. 13, computed for three different choices of the sampling period $\delta t$, with filtered and unfiltered transients measured over (a) phase $c$ and (b) phase $a$. Results obtained using the synthetic transient $v_{o}(t)$ defined in (23), filtering the ground-mode contribution, are marked by circles, computed for transients sampled with $\delta t=1 \mu \mathrm{s}$.

unfiltered transients, and a marginal one in case of a first-order filter. On the contrary, the spatial resolution for a secondorder filter is significantly improved by a higher sampling rate. These observations equally apply to results obtained by monitoring phase $c$, where the fault occurs, or phase $a$. These results are consistent with those presented in Fig. 4, confirming the effectiveness of applying the inverse filters introduced in Sec. III and the practical impact they have on the ability to accurately locate a fault along either single- or multi-conductor power transmission lines.

Protection systems where all three phases are monitored at the same time often filter out the contribution from the ground mode, since it typically presents higher propagation losses. These may reduce the location accuracy for distant faults, while also introducing a dispersive propagation due to their lower propagation speed [28], [40]. The ground mode can be removed by using Clarke transform, combining the three transients $v_{a}(t)$ to $v_{c}(t)$ into a single synthetic transient $v_{o}(t)$

$$
v_{o}(t)=\left[2 v_{a}(t)-(1-\sqrt{3}) v_{b}(t)-(1+\sqrt{3}) v_{c}(t)\right] / 3
$$

equal to the sum of the two aerial-mode signals in Clarke domain. Other linear combinations of the two aerial modes may be chosen, but by virtue of their orthogonality, any choice would yield equivalent access to fault-location information.

The location properties of $v_{o}(t)$ are considered in Fig. 15, when sampling at $1 \mathrm{MHz}$. These results show that when no filter is applied, the correlation for this synthetic transient locates with a lower accuracy than for transients directly measured at single phases $a$ and $c$, which still include the ground mode. With the first-order filter (13) the location accuracy of aerial modes converges to that obtained with single-phase transients. Conversely, the second-order filter presents a significant improvement with respect to transients measured over phase $c$, where the fault occurs, as visible in Fig. 15(a), but no improvement for phase $a$ in Fig. 15(b). Surge-compression therefore appears to take better advantage of the fault-related information provided by aerial modes,

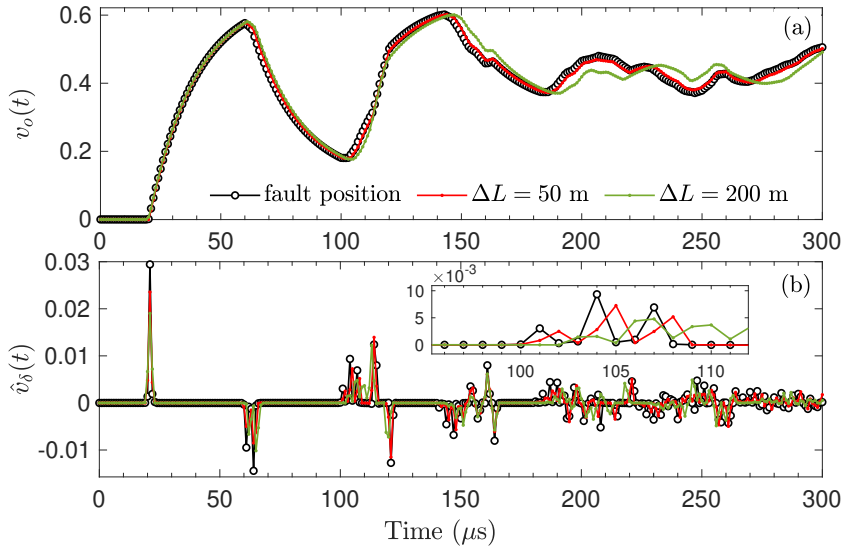

Fig. 16. Comparison between transients simulated at three test positions for: (a) unfiltered and (b) surge-compressed transients. Results based on the synthetic transient $v_{o}(t)$ associated to the transients in Fig. 14, for a $10 \Omega$ PG fault and a sampling rate equal to $1 \mathrm{MHz}$.

compared to using unfiltered transients. Results in the rest of the paper are based on aerial modes only, apart where otherwise stated, as this is a common choice for three-phase lines.

The mechanism behind the higher location accuracy obtained with the proposed method is clarified by Fig. 16, where unfiltered and surge-compressed transients are shown for the actual fault position $L=6 \mathrm{~km}$ and when testing candidate positions 50 and $200 \mathrm{~m}$ further along the line. The three transients in Fig. 16(a) are very similar, resulting in a correlation function equal to 0.98 for a location $200 \mathrm{~m}$ away from the actual fault. Such high values of correlation prevent accurately estimating the fault position.

Conversely, for the surge-compressed transients in Fig. 16(b), the surge echoes have a highly compact support, and a wealth of finer details become apparent, in the shape of a complex sequences of surge echoes. When testing a position $50 \mathrm{~m}$ further from the actual fault, the measured and reference transients have already significantly diverged, resulting in a correlation equal to 0.79 . At $200 \mathrm{~m}$, this value decreases to 0.43 , thus clearly pinpointing the fault position.

A detail in Fig. 16(b) is worth mentioning. The first surge echo expected around $60 \mu \mathrm{s}$ is actually split into two echoes, whose times of arrival closely agree with those expected for aerial and ground modes. Since these results were obtained by filtering out the ground mode, it could appear as contradictory to observe ground-mode related contributions. In fact, it was proven in [25] that PG faults act as modal couplers, thus scattering energy among ground and aerial modes at each interaction with the fault. The increasing number of echoes grouped into clusters in Fig. 16(b) are a consequence of this phenomenon.

\section{B. Fault distance}

The advantages of surge compression were verified when the same $10 \Omega$ PG fault occurs at a closer distance equal to 2 $\mathrm{km}$ and at $18 \mathrm{~km}$. The correlation function was computed also in this case for tested positions $\pm 1 \mathrm{~km}$ around the nominal 


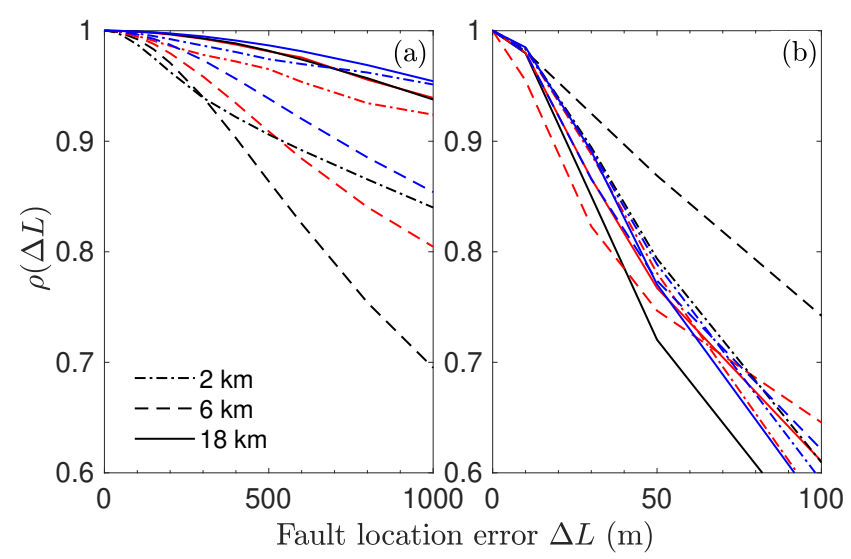

Fig. 17. Correlation function for a $10 \Omega$ PG fault at three positions, for (a) unfiltered and (b) surge-compressed transients. Results obtained from transients at phase $a$ (red curves), phase $c$ (black curves) and synthetic transient $v_{o}(t)$, only including aerial-mode contributions (blue curves). Sampling rate equal to $1 \mathrm{MHz}$.

fault positions. Three sets of transients were considered, using data recorded over phase $a, c$ and using the synthetic transient defined in (23), based only on aerial modes.

Fig. 17 compares the results obtained for these different configurations, for unfiltered and surge-compressed transients. The main feature standing out in these results is the wild variability of the location accuracy for the case of unfiltered transients, ranging from a few hundred meters to beyond one kilometer, before the correlation drops below a 0.9 threshold. On the other hand, results based on the use of the second-order inverse filter present a much more limited variation, mostly within 30-35 m.

In this respect, surge compression appears to provide a more stable performance, less dependent on the fault position, in particular when using only aerial-mode contributions.

\section{High-impedance $P G$ faults and other fault types}

As discussed in Sec. III-C, lower reflections from a line terminations, as well as from a fault, result in a dampening in the line resonances, and ultimately in a loss of spatial resolution for TBM, including correlation. Theoretical models helping to explain how the location accuracy of the correlation metric depends on a line's characteristics have been introduced in [22]; fault-related dissipation in three-phase lines was described in [25] for PG faults. These references provide insights in the physical mechanisms behind spatial resolution, but limited to the level of individual line resonances, rather than the wide-band scenario discussed throughout this paper.

The location performance obtained for a PG fault was therefore tested by running further EMTP simulations considering increasing values of the PG fault impedance, in order to assess the ability of the proposed method to locate higher-impedance faults. These new simulations include fault impedances equal to 100,500 and $1000 \Omega$, as typical values found in the literature [11], [26], [41].

Fig. 18 shows how the correlation fares for higherimpedance PG faults. For unfiltered transients, moving from a

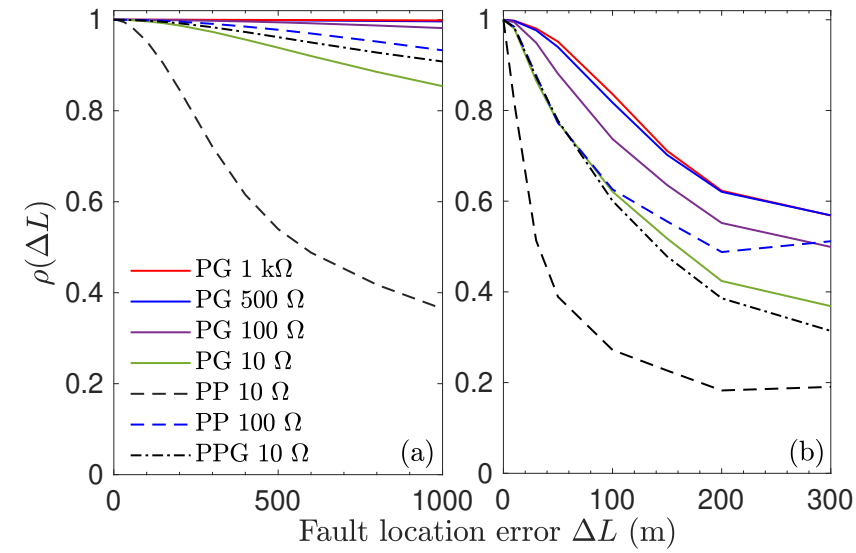

Fig. 18. Correlation function for unfiltered (a) and surge-compressed transients (b), for different faults at $L=6 \mathrm{~km}$. Results obtained using the synthetic transient $v_{o}(t)$ sampled at $1 \mathrm{MHz}$.

$10 \Omega$ fault to a $100 \Omega$ fault results in a sharp loss of resolution, hampering the ability to locate this kind of faults.

The results obtained with surge-compressed echoes shown in Fig. 18(b) are in stark contrast, with a much more limited loss of accuracy. A drop of correlation to 0.9 is observed when testing $100 \Omega$ faults $46 \mathrm{~m}$ away from the actual fault position, compared to a minimum distance of $27 \mathrm{~m}$ for a $10 \Omega$ PG fault. For higher impedances of 500 and $1000 \Omega$, this distance is limited to $75 \mathrm{~m}$.

Other fault types were also considered: a phase-to-phase (PP) fault between phases $b$ and $c$ with an impedance equal to 10 and $100 \Omega$ and a two-phase-to-ground (PPG) shunting phases $b$ and $c$ to the ground through a $10 \Omega$ impedance. Results in Fig. 18 attest to the higher resolution afforded for a $10 \Omega$ PP fault: $156 \mathrm{~m}$ for unfiltered transients and 6 $\mathrm{m}$ when surge-compressed . A higher impedance of $100 \Omega$ drastically affects the accuracy for unfiltered transients, which now exceeds $1 \mathrm{~km}$, while surge-compressed correlation drops to 0.9 within $26 \mathrm{~m}$.

The PPG $10 \Omega$ fault displays no significant difference compared to the respective PG fault for surge-compressed transients. On the other hand, unfiltered transients yield a resolution at 0.9 correlation close to $1 \mathrm{~km}$, compared to the $709 \mathrm{~m}$ resolution reported for the PG fault.

The much higher resolution of the PP fault compared to the PG fault can be better understood by comparing the transients shown in Fig. 19 with those in Fig. 16. The surge-compressed transients for the PP fault feature a single pulse for each echo, since the PP fault does not excite the ground mode. As a result, testing a fault position $50 \mathrm{~m}$ away from the fault yields a significantly different transients, with echoes no longer overlapping with the actual fault transient. Conversely, the PG transient in Fig. 16 is characterized by a sequence of clusters of pulses, increasing in number after each interaction with the fault. The different propagation speeds for ground and aerial modes therefore result in a partial overlapping between the transients even when testing fault positions different from the actual one, hence a slower decay of the correlation function and a partially reduced location accuracy. 

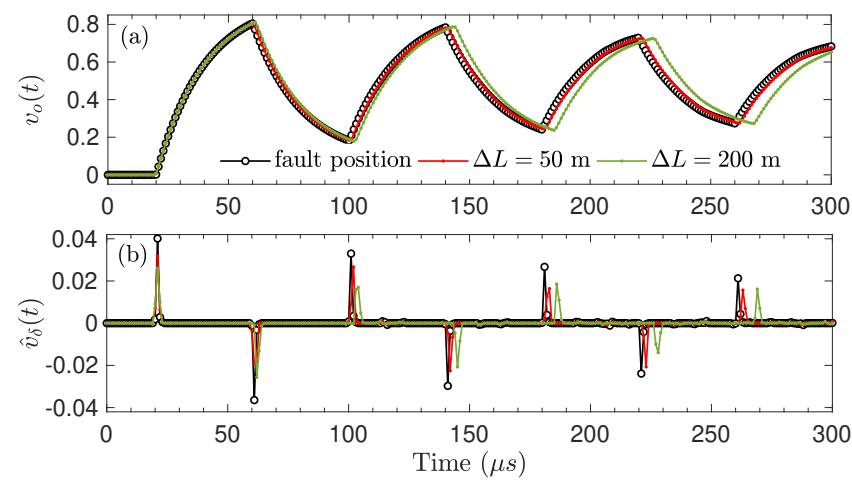

Fig. 19. Comparison between $v_{o}(t)$ transients simulated for three test positions for a $10 \Omega$ PP fault, for: (a) unfiltered and (b) surge-compressed transients. Sampling rate equal to $1 \mathrm{MHz}$.

\section{Surge rise time}

All previous results were based on the fault surge signal having a rise time $T_{r}=20 \mu \mathrm{s}$. The very idea of surge compression is to create transients that no longer depend on the shape of the surge signal. Hence, the correlation function for surge-compressed transients should ideally be independent of the surge rise time. Conversely, when $T_{r}$ increases unfiltered transients present smoother transitions. In contrast to the sharp edges expected for $T_{r}<\delta t$, smoother transitions lead to a slower decay of the correlation function.

Fig. 20(a) presents the correlation function for unfiltered transients with a varying surge rise time, obtained for a $10 \Omega$ PG and PP faults, both at $6 \mathrm{~km}$ from the probed end. These results confirm a significantly higher loss of resolution for $T_{r}$ passing from 10 to $50 \mu \mathrm{s}$ than passing from 1 to $10 \mu \mathrm{s}$, explained by increasingly smooth transitions.

Results for the case of surge-compressed transients are shown in Fig. 20(b) to be practically independent from the surge rise time. These results confirm the effectiveness of the proposed procedure.

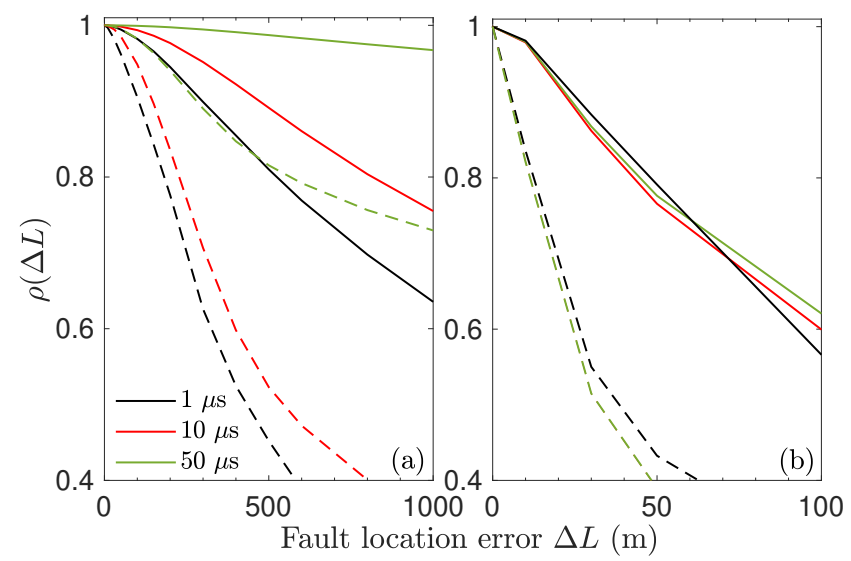

Fig. 20. Correlation function for unfiltered (a) and surge-compressed transients (b), for different values of the surge rise time $T_{r}$, for a PG fault (solid curves) and a PP fault (dashed curves), both at $6 \mathrm{~km}$ with a $10 \Omega$ impedance. Sampling rate equal to $1 \mathrm{MHz}$.

\section{DISCUSSIONS AND CONCLUSION}

The idea of surge-compressing fault transients by means of inverse filters was proven to hold a promising potential for correlation-based fault location, significantly improving the location accuracy, even at sampling rates as low as $100 \mathrm{kHz}$.

These improvements are accessible only if the measured and reference transients are precisely synchronized, before computing their degree of similarity. This procedure should not be confused with transient timestamping as done in multipleended TWM. A novel synchronization procedure was introduced to this end, showing to be practically independent from sampling offsets between measured and reference transients, as opposed to the currently used threshold-based approach, which would otherwise require unnecessarily high speed sampling rates to reduce the impact of sampling offsets.

In this respect, it was shown that increased sampling rates do not result in any improvement in location accuracy when directly computing the correlation between unfiltered transients. Conversely, surge-compressed transients were proven to ensure a spatial resolution steadily improving with the sampling rate. Surge-compression was also shown to be practically independent from the surge rise time and the fault position, ensuring a stable accuracy, whereas unfiltered transients presented wide variations.

When testing high-impedance faults, the direct computation of the correlation between transients was found to be rapidly loosing location accuracy, both for PG and PP faults. In contrast, surge-compression is less affected by high-impedance faults, yielding a location accuracy that even in the case of a $1 \mathrm{k} \Omega$ fault impedance (the worst case), is ten times better than the best accuracy afforded with unfiltered transients.

Currently available protection systems would therefore benefit from the use of the proposed location method, which can be applied to any similarity-based approach, such as EMTR or transient correlations, without requiring any modification in measurement systems.

The proposed ideas can be summarized into an algorithm, involving the following steps

1) for each candidate fault position tested, the delay between measured and reference transients is estimated by means of surge-based synchronization (Sec. IV-B)

2) delay compensation is applied to the reference transient (Sec. IV-C)

3) the transients are surge-compressed applying a secondorder inverse filter (Sec. III)

4) the correlation between the two filtered transients is finally computed

These steps involve simple and numerically efficient operations, calling for matrix multiplications for the surge compression and delay compensation, and a linear least-square fit when estimating the surge-front inception delay.

Future work will focus on optimal inverse-filtering strategies in case of highly dispersive propagation losses, since in this case surge echoes would be increasingly distorted as they propagate along the line, amounting to surge echoes with a rise time increasing over time. This kind of filters would hold the potential for extending the benefits of surge-compressed 
correlation-based fault location to the case of faults occurring at more than $100 \mathrm{~km}$ along long-distance power transmission lines.

\section{REFERENCES}

[1] A. Greenwood, Electrical transients in power systems. John Wiley and Sons Inc., New York, USA, 1991.

[2] Z. Q. Bo, G. Weller, and M. A. Redfern, "Accurate fault location technique for distribution system using fault-generated high-frequency transient voltage signals," IEE Proceedings - Generation, Transmission and Distribution, vol. 146, no. 1, pp. 73-79, Jan 1999.

[3] G. B. Ancell and N. C. Pahalawaththa, "Maximum likelihood estimation of fault location on transmission lines using travelling waves," IEEE Trans. Power Del., vol. 9, no. 2, pp. 680-689, April 1994.

[4] A. Borghetti, M. Bosetti, C. A. Nucci, M. Paolone, and A. Abur, "Integrated use of time-frequency wavelet decompositions for fault location in distribution networks: Theory and experimental validation," IEEE Trans. Power Del., vol. 25, no. 4, pp. 3139-3146, Oct 2010.

[5] R. J. Hamidi and H. Livani, "Traveling-wave-based fault-location algorithm for hybrid multiterminal circuits," IEEE Trans. Power Del., vol. 32, no. 1, pp. 135-144, Feb 2017.

[6] Y. Xi, Z. Li, X. Zeng, X. Tang, X. Zhang, and H. Xiao, "Fault location based on travelling wave identification using an adaptive extended kalman filter," IET Generation, Transmission Distribution, vol. 12, no. 6 , pp. 1314-1322, 2018.

[7] C. Zhang, G. Song, T. Wang, and L. Yang, "Single-ended traveling wave fault location method in dc transmission line based on wave front information," IEEE Trans. Power Del., vol. 34, no. 5, pp. 2028-2038, Oct 2019.

[8] L. Tang, X. Dong, S. Luo, S. Shi, and B. Wang, "A new differential protection of transmission line based on equivalent travelling wave," IEEE Trans. Power Del., vol. 32, no. 3, pp. 1359-1369, June 2017.

[9] B. Li, M. Lv, B. Li, S. Xue, and W. Wen, "Research on an improved protection principle based on differential voltage traveling wave for VSC-HVDC transmission lines," IEEE Trans. Power Del., pp. 1-1, 2020.

[10] B. Li, Y. Li, J. He, B. Li, S. Liu, B. Liu, and L. Xu, "An improved transient traveling-wave based direction criterion for multi-terminal hvdc grid," IEEE Trans. Power Del., pp. 1-1, 2020.

[11] X. Pei, H. Pang, Y. Li, L. Chen, X. Ding, and G. Tang, "A novel ultrahigh-speed traveling-wave protection principle for vsc-based dc grids," IEEE Access, vol. 7, pp. 119765-119773, 2019.

[12] M. Aurangzeb, P. A. Crossley, and P. Gale, "Fault location on a transmission line using high frequency travelling waves measured at a single line end," in 2000 IEEE Power Engineering Society Winter Meeting. Conference Proceedings (Cat. No.00CH37077), vol. 4, Jan 2000, pp. 2437-2442 vol.4

[13] M. Farshad and J. Sadeh, "A novel fault-location method for HVDC transmission lines based on similarity measure of voltage signals," IEEE Trans. Power Del., vol. 28, no. 4, pp. 2483-2490, Oct 2013.

[14] F. Deng, X. Li, and X. Zeng, "Single-ended travelling wave protection algorithm based on full waveform in the time and frequency domains," IET Gener. Transm. Distrib, vol. 12, no. 15, pp. 3680-3691, 2018.

[15] R. Razzaghi, G. Lugrin, H. Manesh, C. Romero, M. Paolone, and F. Rachidi, "An efficient method based on the electromagnetic time reversal to locate faults in power networks," IEEE Trans. Power Del., vol. 28, no. 3, pp. 1663-1673, 2013.

[16] S. He, A. Cozza, and Y. Xie, "Electromagnetic time reversal as a correlation estimator: Improved metrics and design criteria for fault location in power grids," IEEE Trans. Electromagn. Compat., in press.

[17] Z. Wang, R. Razzaghi, M. Paolone, and F. Rachidi, "Time reversal applied to fault location in power networks: Pilot test results and analyses," International Journal of Electrical Power \& Energy Systems, vol. 114, p. 105382, 2020.

[18] G. W. Swift, "The spectra of fault-induced transients," IEEE Trans. Power App. Syst., vol. PAS-98, no. 3, pp. 940-947, May 1979.

[19] L. U. Iurinic, R. G. Ferraz, and A. S. Bretas, "Characteristic frequency of travelling waves applied for transmission lines fault location estimation," in 2013 IEEE Grenoble Conference, June 2013, pp. 1-5.

[20] Y. Wang, Z. Hao, B. Zhang, and F. Kong, "A pilot protection scheme for transmission lines in VSC-HVDC grid based on similarity measure of traveling waves," IEEE Access, vol. 7, pp. 7147-7158, 2019.

[21] X. Zhang, N. Tai, Y. Wang, and J. Liu, "EMTR-based fault location for DC line in VSC-MTDC system using high-frequency currents," IET Generation, Transmission \& Distribution, vol. 11, no. 10, pp. 24992507, 2016
[22] S. He, A. Cozza, and Y. Xie, "On the spatial resolution of fault location techniques based on full fault transients," IEEE Trans. Power Del., 2019, in press.

[23] K. Tailor and A. Ukil, "Fault detection and locating using electromagnetic time reversal (EMTR) technique for HVDC transmission network," in 2019 Asia-Pacific Power and Energy Engineering Conference. IEEE, 2019.

[24] X. Zhang, N. Tai, X. Zheng, and W. Huang, "Wavelet-based EMTR method for fault location of VSC-HVDC transmission lines," The Journal of Engineering, vol. 2019, no. 16, pp. 961-966, 2019.

[25] A. Cozza, S. He, and Y. Xie, "Impact of propagation losses on fault location accuracy in full transient-based methods," IEEE Trans. Power Del., under review.

[26] A. Ghaderi, H. L. Ginn III, and H. A. Mohammadpour, "High impedance fault detection: A review," Electric Power Systems Research, vol. 143, pp. 376-388, 2017.

[27] A. Gopalakrishnan, M. Kezunovic, S. M. McKenna, and D. M. Hamai, "Fault location using the distributed parameter transmission line model," IEEE Transactions on Power Delivery, vol. 15, no. 4, pp. 1169-1174, Oct 2000.

[28] F. V. Lopes, "Settings-free traveling-wave-based earth fault location using unsynchronized two-terminal data," IEEE Trans. Power Del., vol. 31, no. 5, pp. 2296-2298, Oct 2016.

[29] M. M. Saha, J. J. Izykowski, and E. Rosolowski, Fault location on power networks. Springer Science \& Business Media, 2009.

[30] K. Zhu, W. K. Lee, and P. W. T. Pong, "Non-contact capacitive-couplingbased and magnetic-field-sensing-assisted technique for monitoring voltage of overhead power transmission lines," IEEE Sensors Journal, vol. 17, no. 4, pp. 1069-1083, Feb 2017.

[31] A. Ametani, T. Ono, Y. Honaga, and Y. Ouchi, "Surge propagation on Japanese $500 \mathrm{kV}$ untransposed transmission line," Proceedings of the Institution of Electrical Engineers, vol. 121, no. 2, pp. 136-138, February 1974.

[32] T. Cui, X. Dong, Z. Bo, and A. Juszczyk, "Hilbert-transform-based transient/intermittent earth fault detection in noneffectively grounded distribution systems," IEEE Trans. Power Del., vol. 26, no. 1, pp. $143-$ 151, Jan 2011.

[33] T. Ohtaka, M. Iwata, S.-i. Tanaka, and Y. Goda, "Development of an EMTP simulation model of arcing horns interrupting fault current," IEEE Trans. Power Del., vol. 25, no. 3, pp. 2017-2024, 2010.

[34] M. Korkali, H. Lev-Ari, and A. Abur, "Traveling-wave-based faultlocation technique for transmission grids via wide-area synchronized voltage measurements," IEEE Transactions on Power Systems, vol. 27, no. 2, pp. 1003-1011, 2012.

[35] L. Ning, N. Tai, X. Zheng, W.Huang, and M. H. Nadeem, "Detection and classification of MMC-HVDC transmission line faults based on oneterminal transient current signal," in 2017 IEEE Power Energy Society General Meeting, July 2017, pp. 1-5.

[36] R. Razzaghi, M. Paolone, F. Rachidi, J. Descloux, B. Raison, and N. Retière, "Fault location in multi-terminal HVDC networks based on electromagnetic time reversal with limited time reversal window," in 2014 Power Systems Computation Conference. IEEE, 2014, pp. 1-7.

[37] D. Schlichtharle, Digital filters. Springer, Berlin, 2014.

[38] O. Naidu and A. K. Pradhan, "A traveling wave-based fault location method using unsynchronized current measurements," IEEE Trans. Power Del., vol. 34, no. 2, pp. 505-513, April 2019.

[39] P. C. Hansen, V. Pereyra, and G. Scherer, Least squares data fitting with applications. JHU Press, 2013.

[40] R. Benato, S. D. Sessa, M. Poli, C. Quaciari, and G. Rinzo, "An online travelling wave fault location method for unearthed-operated high-voltage overhead line grids," IEEE Trans. Power Del., vol. 33 , no. 6, pp. 2776-2785, Dec 2018.

[41] V. D. Andrade and E. Sorrentino, "Typical expected values of the fault resistance in power systems," in 2010 IEEE/PES Transmission and Distribution Conference and Exposition: Latin America (T D-LA), Nov 2010, pp. 602-609. 